\title{
Control glicémico de pacientes diabéticos hospitalizados en un Servicio de Medicina Interna
}

\author{
IVÁN SOLÍS ${ }^{1}$, NATALIA HURTADO ${ }^{\mathrm{a}}$, DOMINIQUE DEMANGEL ${ }^{\mathrm{a}}$, \\ CLAUDIA CORTÉS ${ }^{2}$, NÉSTOR SOTO ${ }^{1}$
}

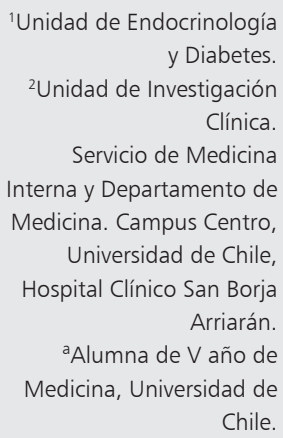

Servicio de Medicina Interna y Departamento de Medicina. Campus Centro,

Universidad de Chile,

Hospital Clínico San Borja Arriarán.

aAlumna de $V$ año de Medicina, Universidad de

Chile.

Recibido el 27 de octubre de 2010, aceptado el 25 de octubre de 2011.

Correspondencia a: Dr. Iván Solís Opazo Unidad de Endocrinología y Diabetes

Departamento de Medicina Interna

Campus Centro,

Universidad de Chile Santa Rosa 1234, Santiago, Chile.

E-mail: ivansolis@med. uchile.cl

\section{Glycemic control in diabetic patients hospitalized in a non-critical care hospital setting}

Background: During hospitalization, hyper and hypoglycemia impairs the prognosis of diabetic patients. Strict glycemic control improves survival in intensive care units. There is no evidence to support it for patients in non-critical wards. Aim: To evaluate the glycemic control of diabetic patients in a non-critical medical unit, and estimate its effect on hospitalization and survival. Material and Methods: Prospective study of all patients admitted to a non-critical ward with a fasting blood glucose (BG) $>126 \mathrm{mg} / \mathrm{dl}$ or $>200 \mathrm{mg} / \mathrm{dl}$ at any time, and patients with known diabetes. Age, sex, type of diabetes, time since diagnosis, chronic complications, prior treatment, length of stay, admission and discharge diagnosis were registered. All capillary BG levels obtained from each patient until discharge, death or transfer, were registered. Results: Ninety nine patients aged $63 \pm 13.4$ years ( 42 males,) were included. Ninety one percent had a type 2 diabetes with a mean duration of 13.8 years. Mean hospital stay was 10.9 days. At least one hypoglycemia below $70 \mathrm{mg} / \mathrm{dl}$ occurred in $21 \%$ of patients and 39.4\% had at least one episode with blood glucose over $300 \mathrm{mg} / \mathrm{dl}$. Median hospital stay of patients with no episode of $B G>200 \mathrm{mg} / \mathrm{dl}$ was 6 days, 10.5 days among patients with at least one episode of $B G>300 \mathrm{mg} / \mathrm{dl}$ and 13 days among patients that had at least one episode of hypoglycemia $(p=0.02)$. Diabetes lasted nine years more among the latter $(p<0.01)$. Three patients that suffered hypoglycemia and two in the rest of the groups, died (NS). Conclusions: Two of three diabetic patients admitted to our non-critical medical ward have a non-optimal glycemic control. Appearance of hypoglycemia is associated with a longer hospital stay.

(Rev Med Chile 2012; 140: 66-72).

Key words: Diabetes mellitus; Health facilities; Hyperglycemia; Hypoglycemia; Inpatients.
4 n Chile, la prevalencia de diabetes ha aumentado desde 6,3 a 9,4\% de la población, y en el grupo de 65 años y más, subió de 15,2 a 26\% desde el año 2003 al 2009 de acuerdo la Encuesta Nacional de Salud ${ }^{1,2}$. En el sistema público, durante el año 2008 se registraron 18.763 egresos hospitalarios por diabetes mellitus en pacientes de 20 años y más, de los cuales 8.921 (47,5\%) fueron en el grupo de mayores de 65 años; en promedio se hospitalizaron durante 11 días $^{3}$. En el Servicio de Medicina de nuestro hospital, la edad promedio de nuestros pacientes es de 64 años, y la prevalencia de diabetes en nuestros hospitalizados alcanza a 22,5\% (Solís I, et al. Prevalencia de Diabetes tipo 2 en pacientes hospitalizados en el Servicio de Medicina Interna. Hospital Clínico San Borja Arriarán. XXIX Congreso Chileno de Medicina Interna, Santiago de Chile, 2007). Esta proporción es coincidente con la descrita por otros autores ${ }^{4,5}$ y en el sistema público nacional ${ }^{6}$. En los pacientes 
diabéticos se invierte el doble de los recursos que en los no diabéticos en cuanto a farmacia, insumos y recursos de personal involucrados ${ }^{7}$. Durante la hospitalización, la hiper e hipoglicemia deterioran la evolución y el pronóstico de pacientes diabéti$\cos ^{8-10}$; más aun, la hiperglicemia de estrés en individuos sin antecedentes de diabetes en unidades de pacientes críticos es claramente deletérea ${ }^{11}$. El control glicémico estricto ha demostrado mejorar la sobrevida y disminuir la morbimortalidad de pacientes diabéticos y no diabéticos en tales unidades $^{12-15}$. Pese a lo anterior, los límites del control metabólico se encuentran en revisión ${ }^{16-18}$. A la fecha, no existe evidencia sólida que soporte lo mismo respecto de pacientes hospitalizados en unidades generales, no críticas.

El control glicémico de pacientes hospitalizados en nuestros hospitales públicos es poco conocido, en especial en unidades no críticas. El objetivo del presente estudio es evaluar el control glicémico de los pacientes diabéticos hospitalizados en el Servicio de Medicina del Hospital San Borja Arriarán, y estimar el efecto de éste sobre aspectos de la hospitalización y sobrevida.

\section{Pacientes y Métodos}

Se realizó un estudio prospectivo, longitudinal y descriptivo entre el 27 de abril y el 22 de junio, 2009. El Hospital Clínico San Borja Arriarán es un hospital público general, urbano, docente y asistencial; el Servicio de Medicina Interna contaba al momento del estudio con 166 camas de baja y mediana complejidad, dividido en 5 unidades de hospitalización. En cada una de ellas, se revisó diariamente el registro de cada paciente ingresado hasta 24 horas de su admisión. Se incluyeron en el estudio todos los pacientes con diabetes mellitus conocida previamente, en control ambulatorio o en tratamiento con hipoglicemiantes orales o insulina; a pacientes sin antecedentes diabéticos, con glicemia de ayunas igual o mayor a $126 \mathrm{mg} /$ dl, o superior a $200 \mathrm{mg} / \mathrm{dl}$ en cualquier momento del día. Los datos se obtuvieron de las indicaciones médicas y los registros en la ficha médica o de enfermería. En un formulario ad-hoc se registraron datos demográficos, edad, género, número de ficha, fecha de ingreso y alta, días de estada, diagnósticos de ingreso y egreso, tipo de diabetes, tiempo de evolución de ésta, complicaciones crónicas conocidas y tratamientos previos a la hospitalización. Se registraron todas las glicemias consignadas en la ficha como parte del manejo habitual durante la hospitalización hasta el alta, fallecimiento, o traslado a otra unidad. El valor de la glicemia se obtuvo de sangre capilar con glucómetros estándar Accu-Chek sensor ${ }^{\circledR}$, al lado de la cama del paciente; además, se registró el tratamiento hipoglicemiante indicado, insulina y/o hipoglicemiantes orales. Se obtuvieron los diagnósticos de alta y las causas registradas de fallecimiento, si procedía. Cada diagnóstico se ordenó de acuerdo a la Clasificación Internacional de Enfermedades, Versión 10 (CIE-10), así como la presencia de complicaciones crónicas de diabetes (ver Anexo). El análisis de los datos fue hecho por investigadores que no participaron en la recolección de los mismos.

\section{Análisis estadístico}

Las variables continuas se agruparon de acuerdo a promedios y/o medianas, utilizándose la desviación estándar o el rango intercuartílico como medida de dispersión de acuerdo a cada caso, y se compararon mediante la prueba t de Student. Las variables discretas se analizaron de acuerdo a una tabla de contingencia $2 \times 2$, obteniendo el riesgo relativo (RR), asociado al respectivo intervalo de confianza. Se utilizó el coeficiente de correlación de Pearson con su nivel de significación para evaluar la relación entre variables continuas.

\section{Resultados}

\section{Datos demográficos y antecedentes}

En el período comprendido entre el 27 de abril y el 22 de junio 2009, ingresaron al Servicio de Medicina Interna 604 pacientes; de los cuales 99 $(16,4 \%)$ presentaban hiperglicemia de acuerdo a los criterios de ingreso; 42 hombres y 57 mujeres, de edad (mediana \pm DE) $63 \pm 13,4$ años (rango intercuartílico [IQR], 57,5 a 71,5). Noventa eran pacientes diabéticos conocidos tipo $2(91 \%)$ y 3 tipo $1(3 \%)$. Seis $(6 \%)$ pacientes tuvieron hiperglicemia al ingreso, no teniendo historia previa de diabetes. Cuarenta y ocho pacientes tenían diez o más años de evolución de su enfermedad, con una mediana de $9 \pm 11,3$ años (IQR, 2-18,3). Sesenta y ocho pacientes $(73,1 \%)$ presentaba al menos una complicación micro o macrovascular; de ellas, las más frecuentes fueron nefropatía diabética y las alteraciones circulatorias periféricas. En relación 
Control glicémico en diabéticos hospitalizados en camas no críticas - I. Solís et al

al tratamiento hipoglicemiante ambulatorio previo a su hospitalización, 29\% de los pacientes eran usuarios de insulina, y 50,5\% usaba hipoglicemiantes orales: de ellos 25 (53,2\%) usaban metformina, 18 (38,3\%) glibenclamida, y 4 otros hipoglicemiantes. Nueve pacientes usaban combinación de sulfonilureas y metformina (Tabla 1).

\section{Control glicémico durante la hospitalización}

Los pacientes se hospitalizaron en promedio durante 10,95 $\pm 8,3$ días. La mediana de glicemia al ingreso fue de $161 \pm 70,5 \mathrm{mg} / \mathrm{dl}$ (IQR, 106,5-215,5). En cuanto al control glicémico intrahospitalario, la mediana de glicemias capilares del grupo general fue

Tabla 1. Características clínicas de pacientes estudiados

\begin{tabular}{|c|c|}
\hline n, género (F/M) & $57 / 42$ \\
\hline Edad, años (IQR) & $63 \pm 13,4(57,5-71,5)$ \\
\hline \multicolumn{2}{|l|}{ Tipo de Diabetes, n (\%) } \\
\hline $\begin{array}{l}\text { Tipo } 2 \\
\text { Tipo } 1 \\
\text { Sin antecedentes* }\end{array}$ & $\begin{array}{rr}90 & (91) \\
3 & (3) \\
6 & (6)\end{array}$ \\
\hline $\begin{array}{l}\text { Evolución, años (IQR) } \\
\geq 10 \text { años evolución, n (\%) }\end{array}$ & $\begin{array}{l}9 \pm 11,1(2-18,3) \\
48(51.6)\end{array}$ \\
\hline \multicolumn{2}{|l|}{ Complicaciones Crónicas n (\%) } \\
\hline $\begin{array}{l}\text { Microvasculares } \\
\text { Nefropatía diabética } \\
\text { Retinopatía diabética } \\
\text { Neuropatía }\end{array}$ & $\begin{aligned} 28 & (30,1) \\
16 & (17,2) \\
1 & (1)\end{aligned}$ \\
\hline $\begin{array}{l}\text { Macrovasculares } \\
\text { Circulatorias periféricas } \\
\text { IAM } \\
\text { ACV } \\
\text { Amputaciones }\end{array}$ & $\begin{aligned} 19 & (20,4) \\
1 & (1) \\
1 & (1) \\
2 & (2,1)\end{aligned}$ \\
\hline $\begin{array}{l}\text { Tratamiento } \\
\text { Hipoglicemiantes orales } \\
\text { Metformina } \\
\text { Glibenclamida } \\
\text { Otras sulfonilureas } \\
\text { Combinación metformina }+ \text { SU } \\
\text { Insulinas } \\
\text { NPH } \\
\text { NPH + Regular }\end{array}$ & $\begin{array}{l}47(50,5)^{\uparrow} \\
25(53,2)^{\S} \\
18(38,3)^{\S} \\
4(8,5)^{\S} \\
9(19,1) \\
27(29)^{\uparrow} \\
24 \\
3\end{array}$ \\
\hline \multicolumn{2}{|c|}{$\begin{array}{l}\text { Valores expresados en n y porcentajes del total, y mediana } \pm \text { des- } \\
\text { viación estándar cuando corresponde. IQR, rango intercuartílico; } \\
\text { IAM, infarto agudo al miocardio; ACV, accidente cerebrovascular; } \\
\text { NPH, Neutral protamine Hagedorn; SU, sulfonilureas. *Pacientes } \\
\text { sin diagnóstico de diabetes previo a la actual hospitalización. 'Cal- } \\
\text { culado en base a pacientes con Diabetes conocida previamente } \\
\text { (n = 93). }{ }^{\S} \text { calculado en relación al subgrupo de pacientes con } \\
\text { hipoglicemiantes orales. }\end{array}$} \\
\hline
\end{tabular}

de $152 \pm 43,9 \mathrm{mg} / \mathrm{dl} ; 66,6 \%$ de los pacientes tuvo al menos 1 episodio de glicemias superior a $200 \mathrm{mg} / \mathrm{dl}$, y 42,4\% de ellos sobre $300 \mathrm{mg} /$ dl. El 21,2\% de los pacientes tuvo al menos un episodio de hipoglicemia bajo $70 \mathrm{mg} / \mathrm{dl}$ (Figura 1). Al agrupar a los pacientes de acuerdo a si presentaban al menos un episodio de hiper o hipoglicemia durante su hospitalización, aquellos que no tuvieron episodios de glicemia superior a $200 \mathrm{mg} / \mathrm{dl}$ tuvieron una mediana de 6 días de hospitalización, en cambio los pacientes con al menos un episodio de hiperglicemia sobre $300 \mathrm{mg} / \mathrm{dl}$ tuvieron una hospitalización más prolongada (mediana 10,5 días, $\mathrm{p}=0,19$ relación al primer grupo). Veintiún pacientes presentaron uno o más episodios de hipoglicemia $<70 \mathrm{mg} / \mathrm{dl}$, lo cual determina $3,4 \%$ de incidencia de ésta en el grupo total (21 de 604 ingresos al servicio en el período); en ellos la mediana de hospitalización fue 13 días ( $\mathrm{p}=$ 0,02 en relación al primer grupo) (Figura 2).

Al comparar a los pacientes que presentaron hipoglicemias durante su hospitalización con aquellos que no la hicieron, los primeros mostraron una tendencia no significativa a tener mayor edad, y en promedio tenían 9 años más de evolución de su enfermedad $(\mathrm{p}=0,0002)$; la dosis promedio de insulina entre ambos no fue diferente (Tabla 2). La presencia de hipoglicemia durante la hospitalización está en el límite de la significación estadística en cuanto al aumento de mortalidad intrahospitalaria ( $p$ $=0,06$ ). Existió una clara correlación entre los episodios de hipoglicemia y número de días de hospitalización (coeficiente de correlación de Pearson, $0,545 \mathrm{p}=0,01)$ pero no con la dosis de insulina promedio diaria administrada, la edad de los pacientes ni los años de evolución de la enfermedad (datos no mostrados); por otro lado, de acuerdo al presente estudio, los episodios de hiperglicemia mayor a $300 \mathrm{mg} / \mathrm{dl}$ no aumentaron significativamente el riesgo de mortalidad $(\mathrm{p}=0,39)$.

Del total de pacientes, setenta y ocho $(78,8 \%)$ usaron insulina durante la hospitalización; de ellos, 28 (36\%) usaron sólo insulina cristalina cada 6 horas, sin insulina basal. No se encontró asociación entre el uso de insulina cristalina en escalas con episodios de hipoglicemia $(p=0,59)$, ni en la mortalidad hospitalaria $(\mathrm{p}=0,13)$. 

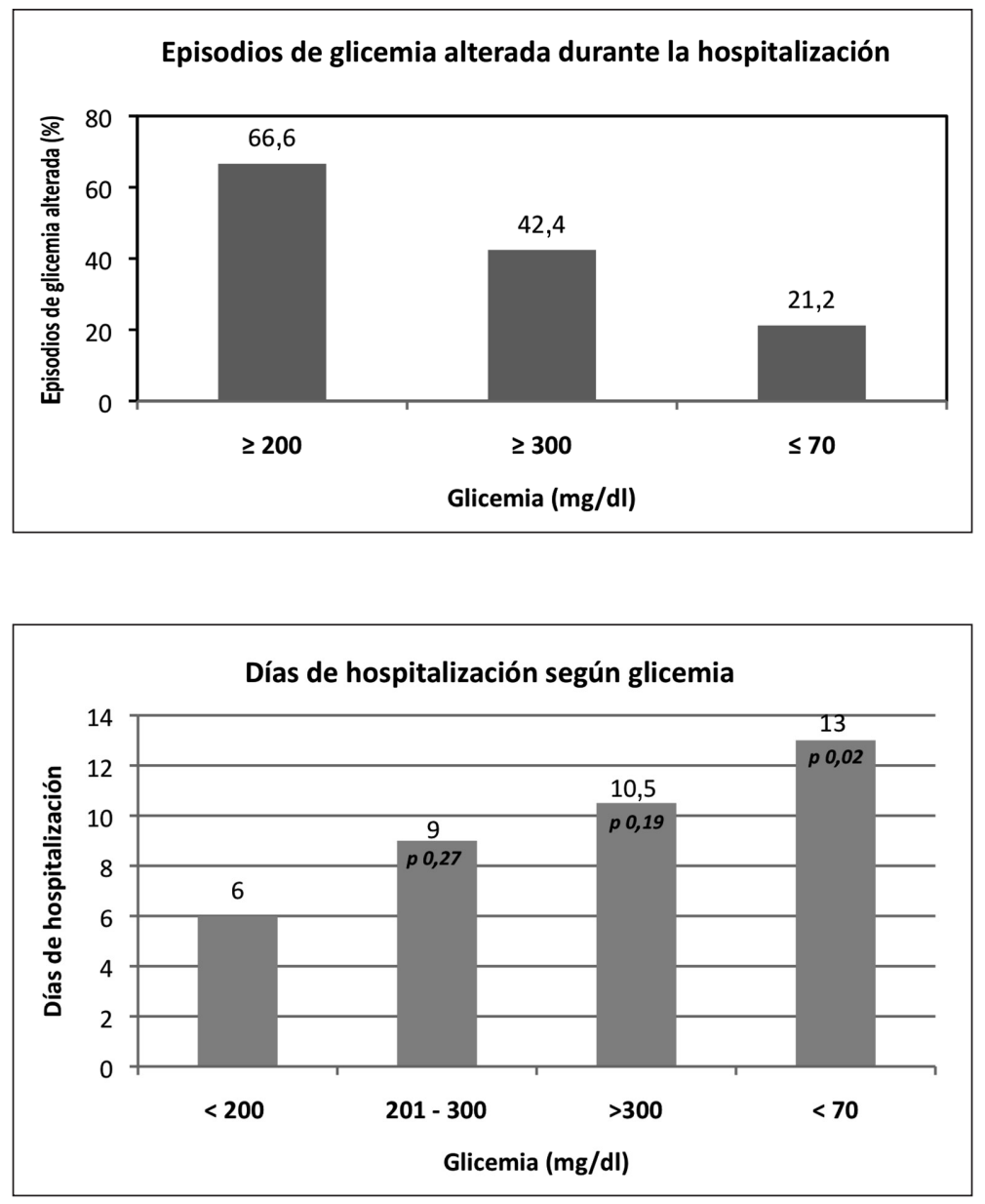

Figura 1. Porcentaje de pacientes con al menos un episodio de glicemia alterada durante la hospitalización.

Figura 2. Mediana de días de hospitalización en pacientes con al menos un episodio de glicemia alterada, de acuerdo a criterios descritos en Figura 1.

Tabla 2. Mortalidad y factores asociados a hipoglicemia intrahospitalaria

\begin{tabular}{|c|c|c|c|c|c|}
\hline & $\begin{array}{c}\text { Hipoglicemia } \\
n=21\end{array}$ & $\begin{array}{l}\text { No Hipoglicemia } \\
\qquad n=78\end{array}$ & $\mathbf{p}$ & $\mathbf{R R}$ & IC $95 \%$ \\
\hline Edad (años) & $64,9 \pm 15,1$ & $62,9 \pm 12,9$ & 0,54 & & \\
\hline Evolución (años) & $21 \pm 13,1$ & $12 \pm 9,9$ & $<0,001$ & & $3,89-14,19$ \\
\hline Hospitalización (días) & $16,7 \pm 10,9$ & $9,38 \pm 6,75$ & $<0,001$ & & $3,57-11,18$ \\
\hline $\begin{array}{l}\text { Nefropatía } \\
\text { No nefropatía }\end{array}$ & $\begin{array}{r}5 \\
16\end{array}$ & $\begin{array}{l}16 \\
62\end{array}$ & 0,73 & 1,16 & $0,48-2,8$ \\
\hline $\begin{array}{l}\mathrm{HGO} \\
\mathrm{No} \mathrm{HGO}\end{array}$ & $\begin{array}{r}5 \\
16\end{array}$ & $\begin{array}{l}24 \\
54\end{array}$ & 0,55 & 0,75 & $0,3-1,86$ \\
\hline $\begin{aligned} \text { Edad } & \geq 75 \text { años } \\
& <75 \text { años }\end{aligned}$ & $\begin{array}{r}6 \\
15\end{array}$ & $\begin{array}{l}13 \\
65\end{array}$ & 0,24 & 1,68 & $0,75-3,7$ \\
\hline $\begin{array}{l}\text { Fallecidos } \\
\text { No Fallecidos }\end{array}$ & $\begin{array}{r}3 \\
18\end{array}$ & $\begin{array}{r}2 \\
76\end{array}$ & 0,06 & 5,57 & $0,99-31,2$ \\
\hline
\end{tabular}

Variables continuas en promedio \pm DE. HGO: hipoglicemiantes orales durante la hospitalización. RR: riesgo relativo; IC: intervalo de confianza. Se considera significativo $p<0,05$. 
Control glicémico en diabéticos hospitalizados en camas no críticas - I. Solís et al

\section{Diagnósticos de egreso}

Se registraron 233 diagnósticos de egreso en 99 pacientes, en promedio 2,3 por paciente; al excluir diabetes $(n=74)$ e hipertensión arterial $(n=54)$ -ciertamente los más frecuentes- predominan las causas cardio y cerebrovasculares $(18,1 \%)$, seguido de las causas digestivas $(15,5 \%)$, infecciosas $(10,5 \%)$, nefrológicas $(9,5 \%)$ y respiratorias (6,7\%) como razón de la hospitalización (Tabla 3). Dentro de los diagnósticos de egreso cardiovasculares, los más frecuentes son insuficiencia cardiaca y enfermedad coronaria, y en las causas digestivas, la cirrosis hepática. Las infecciones más frecuentes fueron la infección urinaria y la neumonía.

Cinco pacientes fallecieron durante la hospitalización, determinando una mortalidad de $5 \%$ en el grupo estudiado (5/99); tres de ellos presentaron una complicación infecciosa de enfermedades neoplásicas avanzadas (peritonitis difusa secundaria a una perforación de un cáncer de colon, neumonía aspirativa secundaria a una obstrucción intestinal por un cáncer gástrico avanzado, sepsis asociada a una leucemia linfocítica crónica) y una paciente portadora de insuficiencia renal crónica en hemodiálisis presentó un accidente cerebrovascular. En un paciente no fue posible especificar la causa de fallecimiento. De veintiún pacientes en los cuales se registró al menos un episodio hipoglicémico, tres pacientes finalmente fallecieron, determinando una letalidad asociada a hipoglicemia -aunque no atribuida directamente a ésta- de 14,2\%.

\section{Discusión}

La calidad del control glicémico en pacientes diabéticos hospitalizados es uno de los parámetros considerados al evaluar la calidad de la atención en hospitales de Norteamérica ${ }^{19}$; está demostrado que niveles glicémicos adecuados evitan complicaciones y acortan los períodos de hospitalización. El American College of Endocrinology y la American Association of Clinical Endocrinologists (ACE/ AACE) han dado a conocer criterios para un óptimo control glicémico en pacientes hospitalizados, considerando adecuadas glicemias preprandiales inferiores a140 mg/dl e inferiores a $180 \mathrm{mg} / \mathrm{dl}$ en una determinación aleatoria, en pacientes no subsidiarios de cuidados intensivos ${ }^{17}$.

Nuestros resultados exponen una población de pacientes diabéticos hospitalizados de edad avanzada, de más de 10 años de evolución, con
Tabla 3. Diagnósticos de egreso más frecuentes en pacientes diabéticos hospitalizados

\begin{tabular}{|lcc|}
\hline Diagnósticos de egreso & n & \%* \\
Cardiovasculares & 19 & 18,1 \\
Insuficiencia cardiaca & 6 & $(31,5)$ \\
Accidente cerebrovascular & 4 & $(21,0)$ \\
Enfermedad coronaria & 3 & $(15,8)$ \\
Digestivas & 16 & 15,5 \\
Daño hepático crónico & 11 & $(68,7)$ \\
Infecciosas & 11 & 10,5 \\
Infección urinaria & 5 & $(45,5)$ \\
Neumonía & 4 & $(36,4)$ \\
Nefrológicas & 10 & 9,5 \\
Insuficiencia Renal Crónica & 5 & $(50)$ \\
Sd. Nefrótico & 2 & $(20)$ \\
Respiratorias & 7 & 6,7 \\
EPOC - Asma & 5 & $(71,5)$ \\
Otras & 42 & 40 \\
\hline Total & 105 & 100 \\
\hline
\end{tabular}

*Entre paréntesis la frecuencia del diagnóstico en relación al total de su grupo. EPOC: Enfermedad Pulmonar Obstructiva Crónica. Las causas respiratorias no incluyen neumonía.

una alta frecuencia de complicaciones crónicas. Su control glicémico está lejos de ser adecuado de acuerdo a los parámetros preconizados por ACE/ AACE. La hiperglicemia es significativa; dos tercios de los pacientes tienen al menos un episodio de glicemia superior a $200 \mathrm{mg} / \mathrm{dl}$ y uno de cada cinco presenta un episodio de hipoglicemia inferior a $70 \mathrm{mg} / \mathrm{dl}$. Estudios similares publicados muestran cifras comparables a nuestra realidad; Schnniper et al., en un estudio prospectivo de 107 pacientes diabéticos en una unidad de medicina no crítica, publicado el año 2006 , reporta $76 \%$ de pacientes con glicemias sobre $180 \mathrm{mg} / \mathrm{dl}$ y $11 \%$ de pacientes con un episodio de glicemia inferior a $60 \mathrm{mg} / \mathrm{dl}^{20}$; un estudio de 44 hospitales de Estados Unidos de Norteamérica muestra $77 \%$ de pacientes con glicemias superiores a $200 \mathrm{mg} / \mathrm{dl}$, y $12 \%$ de episodios de hipoglicemia bajo $60 \mathrm{mg} / \mathrm{dl}^{21}$. Sin duda, el control glicémico previo a la hospitalización tiene que ver con la evolución del paciente; sin embargo, también se han invocado factores como la inercia clínica, es decir, la falta de toma de decisiones respecto de la hiper o hipoglicemia, a la tolerancia ante cifras elevadas como grandes responsables de esta situación ${ }^{5,22}$. Ya en 2006, la American Diabetes Association llamaba a la acción respecto a 
estos hechos. Nuestro trabajo no profundiza en la toma de decisiones ni en diversos esquemas de tratamiento con insulina, aunque el uso de escalas (sliding scales) de insulina cristalina cada 6 horas es una práctica habitual y muy arraigada en nuestra cultura médica y tiene que ver con grandes fluctuaciones glicémicas y un demostrado mayor riesgo de descontrol metabólico ${ }^{8}$.

De acuerdo a nuestro estudio, 21/604 (3,4\%) de nuestros pacientes presentó al menos un episodio de hipoglicemia durante su hospitalización en el período analizado. La letalidad asociada a ella fue del 14\% (3 de 21), si bien no es posible establecer una causalidad directa, claramente tiene mayor significancia en términos pronósticos. Además de lo mencionado, no logramos identificar en el presente estudio otros factores asociados a hipoglicemia. Turchin et $\mathrm{al}^{10}$, en un estudio de cohorte retrospectivo reporta una frecuencia de $7,7 \%$, definiendo hipoglicemia bajo $50 \mathrm{mg} / \mathrm{dl}$, y de hiperglicemia superior a $300 \mathrm{mg} / \mathrm{dl}$ en $29,1 \%$. Es de notar que, al igual que nuestro estudio, no encuentra asociación de la hipoglicemia con complicaciones crónicas, velocidad de filtración glomerular, función hepática ni edad. En el mismo estudio, los pacientes con hipoglicemia estuvieron 2,8 días más hospitalizados que aquellos que no la presentaron; y describe una asociación entre hipoglicemia y mortalidad intrahospitalaria, hecho que nosotros constatamos pero que no alcanzó significación estadística, probablemente por el limitado tamaño muestral. Kagansky et al ${ }^{9}$ en un estudio de casos y controles en pacientes hospitalizados mayores de 70 años, tomando como hipoglicemia valores inferiores a $60 \mathrm{mg} / \mathrm{dl}$, reporta una frecuencia de hipoglicemias de 5,2\%, cifra más cercana a la nuestra, con una mortalidad intrahospitalaria de $26 \%$ en el grupo afectado. En el estudio no queda claro si también se incluyeron pacientes de cuidados intensivos.

En el presente estudio, la ausencia de asociación con factores reconocidamente influyentes en la aparición de hipoglicemias probablemente se deba al diseño del mismo, no concebido para estudiar específicamente este problema en nuestros pacientes. Por lo mismo, no podemos concluir que la presencia de hipoglicemias sea o no un factor de riesgo independiente para mortalidad. Algunos autores consideran a la hipoglicemia intrahospitalaria un marcador de gravedad y de mala evolución clínica ${ }^{9,10}$.
Los diagnósticos de egreso no difieren de lo presentado por Novik et $\mathrm{al}^{6}$ en un servicio de medicina interna general de un hospital público de nuestro país, ni a lo reportado internacionalmente.

Es de notar que la mortalidad descrita por nuestro estudio en el grupo de pacientes que presentaron hipoglicemia durante la hospitalización, casi triplica (5\% vs $14,2 \%$ ) la del grupo general.

En conclusión, podemos establecer que los pacientes diabéticos hospitalizados en un servicio no intensivo de un hospital público cuentan con un control glicémico deficiente, con altas tasas de hiper e hipoglicemias, y que éstas últimas se relacionan mejor con la mortalidad intrahospitalaria; las causas de egreso siguen siendo principalmente cardiovasculares. Futuros estudios con aplicación de protocolos más estrictos permitirán conocer la mejor forma de tratar a nuestros pacientes diabéticos en unidades no intensivas para obtener las metas de control, acortar las hospitalizaciones y mejorar su pronóstico.

Agradecimientos. A los alumnos de Medicina de la Universidad de Chile que participaron en la recolección de datos, sin su colaboración habría sido imposible la realización del presente estudio.

\section{Referencias}

1. Gobierno de Chile, Ministerio de Salud. Resultados I Encuesta de Salud, Chile 2003. Disponible en epi.minsal. $\mathrm{cl} /$ epi/html/invest/ENS/ENS.htm

2. Gobierno de Chile, Ministerio de Salud. Encuesta Nacional de Salud ENS 2009-2010. Tomo II, Cap. V, Resultados. Disponible en www.encuestasalud.cl/pdf/ InformeENS_2009-2010_CAP5.pdf

3. Gobierno de Chile, Ministerio de Salud. Egresos hospitalarios de ambos sexos según edad y causas. Chile, 2007. Disponible en deis.minsal.cl/egresoshospitalarios

4. Levetan CS, Passaro M, Jablonski K, Kass M, Ratner RE. Unrecognized diabetes among hospitalized patients. Diabetes Care 1998; 21: 246-9.

5. Knecht LA, Gauthier SM, Castro JC, Schmidt RE, Whitaker MD, Zimmerman RS, et al. Diabetes Care in the hospital: Is there clinical inertia? J Hosp Med 2006; 1 (3): 151-60.

6. Novik V, Aninat F, Valderrama H, Cardemil F. Prevalencia de Diabetes Mellitus en un Servicio de Medicina Interna. Causas de hospitalización y factores asociados a morbimortalidad. Rev. Chil Endocrinol Diabetes 2009; 1: 19-23. 
Control glicémico en diabéticos hospitalizados en camas no críticas - I. Solís et al

7. Caporale JE, Calvo H, Gagliardino JJ. Costos de atención médica de personas con diabetes anteriores y posteriores a su hospitalización en Argentina. Rev Panam Salud Publica 2006; 20 (6): 361-8.

8. Umpierrez GE, Palacio A, Smiley D. Sliding scale insulin use: myth or insanity?. Am J Med 2007; 120 (7): 563-7.

9. Kagansky N, Levy S, Rimon E, Cojocaru L, Fridman A, Ozer Z, Knobler H, et al. Hypoglycemia as a predictor of mortality in hospitalized elderly patients. Arch Intern Med 2003; 163 (15): 1825-9.

10. Turchin A, Matheny ME, Shubina M, Scanlon JV, Greenwood B, Pendergrasset ML, et al. Hypoglycemia and clinical outcomes in patients with diabetes hospitalized in the general ward. Diabetes Care 2009; 32 (7): 1153-7.

11. Umpierrez GE, Isaacs SD, Bazargan N, You X, Thaler LM, Kitabchi AE. Hyperglycemia: an independent marker of in-hospital mortality in patients with undiagnosed diabetes J Clin Endocrinol Metab 2002; 87 (3): 978-82.

12. Van Den Berghe G, Wouters P, Weekers F, Verwaest C, Bruyninckx F, Schetz M, et al. Intensive insulin therapy in the critically ill patients. N Engl J Med 2001; 345 (19): 1359-67.

13. Van den Berghe G, Wilmer A, Hermans G, Meersseman W, Wouters PJ, Milants I, et al. Intensive insulin therapy in the medical ICU. N Engl J Med 2006; 354 (5): 449-61.

14. Malmberg K, Rydén L,Wedel H, Birkeland K, Bootsma A, Dickstein K, et al. Intense metabolic control by means of insulin in patients with diabetes mellitus and acute myocardial infarction (DIGAMI 2): effects on mortality and morbidity. Eur Heart J 2005; 26 (7): 650-61.

15. Zerr KJ, Furnary AP, Grunkemeier GL, Bookin S, Kanhe- re V, Starr A. Glucose control lowers the risk of wound infection in diabetics alter open heart operations. Ann Thorac Surg 1997; 63: 356-61.

16. NICE-SUGAR Study Investigators: Finfer S, Chittock DR, Yu-Shuo Su S, Blair D, Foster D, Dhingra V, et al. Intensive versus conventional glucose control in critically ill patients. N Engl J Med 2009; 360 (13): 1283-97.

17. Moghissi E, Korytkowski M, Dinardo M, Einhorn D, Hellman R, Hirsch I, et al. American Association of Clinical Endocrinologists and American Diabetes Association Consensus Statement on Inpatient Glycemic Control. Endocrine practice: official journal of the American College of Endocrinology and the American Association of Clinical Endocrinologists 2009; 1-17.

18. Currie CJ, Peters JR, Tynan A, Evans M, Heine RJ, Bracco $\mathrm{OL}$, et al. Survival as a function of $\mathrm{HbA}(1 \mathrm{c})$ in people with type 2 diabetes: a retrospective cohort study. Lancet 2010; 375 (9713): 481-9.

19. Magee MF. Hospital protocols for targeted glycemic control: Development, implementation, and models for cost justification. American Journal of Health-System Pharmacy 2007; 64 (10 Suppl 6): S15-20; quiz S21-3.

20. Schnipper JL, Barsky EE, Shaykevich S, Fitzmaurice G, Pendergrass ML. Inpatient management of diabetes and hyperglycemia among general medicine patients at a large teaching hospital. J Hosp Med 2006; 1 (3): 145-50.

21. Wexler DJ, Meigs JB, Cagliero E, Nathan DM, Grant RW. Prevalence of hyper- and hypoglycemia among inpatients with diabetes: a national survey of 44 U.S. hospitals. Diabetes Care 2007; 30 (2): 367-9.

22. Umpierrez GE, Maynard G. Glycemic chaos (not glycemic control) still the rule for inpatient care: how do we stop the insanity?. J Hosp Med 2006; 1 (3): 141-4.

\section{Anexo. Criterios diagnósticos para complicaciones crónicas de diabetes}

\section{Retinopatía: según evolución oftalmológica previa reciente en la ficha}

Nefropatía: creatinina mayor a 1,5 mg/dl, sedimento urinario con presencia de cilindros hialinos, proteinuria presente y medida en 24 horas, y/o microalbuminuria superior a 30 mg/g creatinina. Hemodiálisis crónica asociada a Diabetes

Enfermedad coronaria: infarto agudo al miocardio previo demostrado en ECG, estudio invasivo o no invasivo compatible con alteraciones isquémicas del miocardio que conste en la ficha clínica

Compromiso gastrointestinal: elementos de gastroparesia demostrada en estudio digestivo, diarrea crónica

Neuropatía periférica: parestesias en guante y calcetín, EMG y velocidad de conducción y/o evaluación neurológica compatible

Neuropatía autonómica: hipotensión ortostática estudiada, alteraciones de la sudoración

Pié diabético: actual o previo, úlceras, artropatía de Charcot, antecedentes de ella en ficha clínica

Amputación: actual o previa, de causa no traumática.

Por lo menos una de las variables expuestas en cada ítem debe presentarse para asignar el criterio 